International Electronic Journal of Algebra

VOLUME 25 (2019) 186-198

DOI: $10.24330 /$ ieja.504147

\title{
ON THE CLASSICAL PRIME SPECTRUM OF LATTICE MODULES
}

\author{
Pradip Girase, Vandeo Borkar and Narayan Phadatare \\ Received: 3 June 2018; Accepted: 4 November 2018 \\ Communicated by Abdullah Harmancı
}

\begin{abstract}
Let $M$ be a lattice module over a $C$-lattice $L$. A proper element $P$ of $M$ is said to be classical prime if for $a, b \in L$ and $X \in M, a b X \leq P$ implies that $a X \leq P$ or $b X \leq P$. The set of all classical prime elements of $M$, $\operatorname{Spec}^{c p}(M)$ is called as classical prime spectrum. In this article, we introduce and study a topology on $\operatorname{Spec}^{c p}(M)$, called as Zariski-like topology of $M$. We investigate this topological space from the point of view of spectral spaces. We show that if $M$ has ascending chain condition on classical prime radical elements, then $\operatorname{Spec}^{c p}(M)$ with the Zariski-like topology is a spectral space.
\end{abstract}

Mathematics Subject Classification (2010): 06D10, 06E10, 06F10

Keywords: Classical prime element, classical prime spectrum, classical prime radical element, Zariski-like topology, spectral space

\section{Introduction}

Zariski-like topology on the classical prime spectrum of a module is being introduce and studied by M. Behboodi and M. J. Noori in [7]. There are many generalizations of the Zariski topology over the set of all prime submodules of a $R$ module $M$ (see [5], [6], [14]). As a generalization of most of the results in [7], we introduce and study the Zariski-like topology on the classical prime spectrum of a lattice module $M$ over a $C$-lattice $L$.

A lattice $L$ is said to be complete, if for any subset $S$ of $L$, we have $\vee S, \wedge S \in L$. A complete lattice $L$ with least element $0_{L}$ and greatest element $1_{L}$ is said to be a multiplicative lattice, if there is defined a binary operation "." called multiplication on $L$ satisfying the following conditions:

(1) $a . b=b . a$, for all $a, b \in L$;

(2) $a .(b . c)=(a . b) . c$, for all $a, b, c \in L$;

(3) $a .\left(\vee_{\alpha} b_{\alpha}\right)=\vee_{\alpha}\left(a . b_{\alpha}\right)$, for all $a, b_{\alpha} \in L$;

(4) $a .1_{L}=a$, for all $a \in L$.

Henceforth, $a . b$ will be simply denoted by $a b$. 
An element $a$ in $L$ is called compact if $a \leq \vee_{\alpha \in I} b_{\alpha}$ ( $I$ is an indexed set) implies $a \leq b_{\alpha_{1}} \vee b_{\alpha_{2}} \vee \cdots \vee b_{\alpha_{n}}$ for some subset $\left\{\alpha_{1}, \alpha_{2}, \cdots, \alpha_{n}\right\}$ of $I$. By a $C$-lattice, we mean a multiplicative lattice $L$, with least element $0_{L}$ and greatest element $1_{L}$ which is compact as well as multiplicative identity, that is generated under joins by a multiplicatively closed subset $C$ of compact elements of $L$. An element $a \in L$ is said to be proper, if $a<1_{L}$.

A proper element $p$ of a multiplicative lattice $L$ is said to be prime if $a b \leq p$ implies $a \leq p$ or $b \leq p$ for $a, b \in L$. The collection of all prime elements of $L$ is denoted by $\operatorname{Spec}(L)$.

The Zariski topology on the set $\operatorname{Spec}(L)$ of all prime elements in multiplicative lattices is being studied in [21], by Thakare, Manjarekar and Maeda and in [20], by Thakare and Manjarekar as a generalization of the Zariski topology of a commutative ring with unity.

A proper element $m$ of a multiplicative lattice $L$ is said to be maximal if for every $x \in L$ with $m<x \leq 1_{L}$ implies $x=1_{L}$.

A complete lattice $M$ with smallest element $0_{M}$ and greatest element $1_{M}$ is said to be a lattice module over the multiplicative lattice $L$ or $L$-module if there is a multiplication between elements of $M$ and $L$, denoted by $a N \in M$, for $a \in L$ and $N \in M$, which satisfies the following properties:

(1) $(a b) N=a(b N)$;

(2) $\left(\vee_{\alpha} a_{\alpha}\right)\left(\vee_{\beta} N_{\beta}\right)=\left(\vee_{\alpha \beta} a_{\alpha} N_{\beta}\right)$;

(3) $1_{L} N=N$;

(4) $0_{L} N=0_{M}$; for all $a, b, a_{\alpha} \in L$, and for all $N, N_{\beta} \in M$.

Let $M$ be a lattice module over a $C$-lattice $L$. For $N \in M, b \in L$, denote $(N: b)=$ $\vee\{K \in M \mid b K \leq N\}$. If $a, b \in L$, we write $(a: b)=\vee\{x \in L \mid b x \leq a\}$. If $A, B \in M$, then $(A: B)=\vee\{x \in L \mid B x \leq A\}$. An element $N \in M$ is said to be compact if $N \leq \vee_{\alpha \in I} A_{\alpha}$ ( $I$ is an indexed set) implies $N \leq A_{\alpha_{1}} \vee A_{\alpha_{2}} \vee \cdots \vee A_{\alpha_{n}}$ for some subset $\left\{\alpha_{1}, \alpha_{2}, \cdots, \alpha_{n}\right\}$ of $I$.

An element $N \in M$ is said to be meet principal (respectively join principal) if it satisfies the identity $A \wedge a N=(a \wedge(A: N)) N$ (respectively $((a N \vee A): N)=$ $(a \vee(A: N)))$ for all $a \in L$ and for all $A \in M$. Also $N$ is said to be principal if it is both join as well as meet principal. If each element of $M$ is the join of principal (compact) elements of $M$, then $M$ is called the principally generated (compactly generated) lattice module.

An element $N \in M$ is said to be proper, if $N<1_{M}$. A proper element $N$ of a lattice module $M$ is said to be prime if $a X \leq N$ implies $X \leq N$ or $a 1_{M} \leq N$, i.e., $a \leq\left(N: 1_{M}\right)$ for every $a \in L$ and $X \in M$. The prime spectrum of a lattice module 
$M$ is the set of all prime elements of $M$ and it is denoted by $\operatorname{Spec}(M)$. In [4], S. Ballal and V. Kharat studied the Zariski topology over $\operatorname{Spec}(M)$ as a generalization of the results carried out in [20], [21]. Also in [10], F. Callialp et al. studied the Zariski topology over $\operatorname{Spec}(M)$ over multiplicative lattice $L$.

A non-zero element $N \in M$ is said to be second, if for $a \in L$, either $a N=N$ or $a N=0_{M}$. The Zariski topology on the second spectrum of a lattice module is studied by N. Phadatare et al. in [17]. In [18], N. Phadatare and V. Kharat introduced and studied the concept of second radical elements of a lattice module $M$ over a $C$-lattice $L$ as a generalization of second socle of a submodule of $R$-module $M$. An element $N<1_{M}$ of $M$ is said to be maximal if $N \leq B$ implies either $N=B$ or $B=1_{M}, B \in M$. A non-zero element $K \neq 1_{M}$ of $M$ is said to be minimal if $0_{M} \leq N<K$ implies $N=0_{M}, N \in M$.

Further all these concepts and for more information on multiplicative lattices, lattice modules and topology, the reader may refer ([1], [2], [13], [16], [19]).

\section{Zariski-like topology on $\operatorname{Spec}^{c p}(M)$}

Let $M$ be a lattice module over a $C$-lattice $L$. A proper element $P \in M$ is said to be classical prime if for $a, b \in L$ and $X \in M, a b X \leq P$ implies that $a X \leq P$ or $b X \leq P([15])$. The classical prime spectrum $\operatorname{Spec}^{c p}(M)$ is defined to be the set of all classical prime elements of $M$, i.e., $\operatorname{Spec}^{c p}(M)=\{P \in M \mid P$ is a classical prime element of $M$ \}. Let $N$ be any element of $M$. Let $F^{c}(N)$ be the set of all classical prime elements of $M$ which contains N, i.e., $F^{c}(N)=\left\{P \in \operatorname{Spec}^{c p}(M) \mid N \leq P\right\}$. Note that, $F^{c}\left(0_{M}\right)=\operatorname{Spec}^{c p}(M)$ and $F^{c}\left(1_{M}\right)$ is an empty set.

Proposition 2.1. Let $M$ be a lattice module over a $C$-lattice $L$ and $N, N_{i}, K \in M$ $(i \in I)$. Then the following statements holds.

(1) $\cap_{i \in I} F^{c}\left(N_{i}\right)=F^{c}\left(\vee_{i \in I} N_{i}\right)$ for any index set $I$.

(2) $F^{c}(N) \cup F^{c}(K) \subseteq F^{c}(N \wedge K)$.

Proof. (1) Since for each $i \in I, N_{i} \leq \vee N_{i}$, therefore for $P \in F^{c}\left(\vee_{i \in I} N_{i}\right)$, we have $\vee_{i \in I} N_{i} \leq P$ and hence $N_{i} \leq P$ and $P \in F^{c}\left(N_{i}\right)$. Therefore $F^{c}\left(\vee_{i \in I} N_{i}\right) \subseteq$ $\cap_{i \in I} F^{c}\left(N_{i}\right)$.

Conversely, suppose that $P \in \cap_{i \in I} F^{c}\left(N_{i}\right)$, then $P \in F^{c}\left(N_{i}\right)$ for each $i \in I$, therefore $N_{i} \leq P$ for each $i \in I$. Hence $\vee_{i \in I} N_{i} \leq P$ and so $P \in F^{c}\left(\vee_{i \in I} N_{i}\right)$. Consequently, $\cap_{i \in I} F^{c}\left(N_{i}\right) \subseteq F^{c}\left(\vee_{i \in I} N_{i}\right)$. Thus $\cap_{i \in I} F^{c}\left(N_{i}\right)=F^{c}\left(\vee_{i \in I} N_{i}\right)$.

(2) Since $N \wedge K \leq N, K$, we have $F^{c}(N), F^{c}(K) \subseteq F^{c}(N \wedge K)$ and therefore $F^{c}(N) \cup F^{c}(K) \subseteq F^{c}(N \wedge K)$. 
Let $\xi^{c}(M)=\left\{F^{c}(N) \mid N \in M\right\}$, then $\xi^{c}(M)$ contains empty set and $\operatorname{Spec}^{c p}(M)$. By Proposition 2.1, $\xi^{c}(M)$ is closed under arbitrary intersections. In general $\xi^{c}(M)$ is not closed under finite union. A lattice module $M$ over a $C$-lattice $L$ is called top lattice module if $\xi^{c}(M)$ is closed under finite union. In this case, $\xi^{c}(M)$ induces a topology $\tau^{c}$ on $\operatorname{Spec}^{c p}(M)$, we call it the Zariski topology.

Let $M$ be a lattice module over a $C$-lattice $L$. For each element $N$ of $M$, let $G^{c}(N)=\operatorname{Spec}^{c p}(M)-F^{c}(N)$ and $\mathbb{G}^{c}(N)=\left\{G^{c}(N) \mid N \in M\right\}$. Let $\psi^{c}(M)$ be the collection of all unions of finite intersections of elements of $\mathbb{G}^{c}(N)$, then $\psi^{c}(M)$ is a topology on $\operatorname{Spec}^{c p}(M)$ by the sub-basis $\mathbb{G}^{c}(N)$. We call the topology $\psi^{c}(M)$, a Zariski-like topology.

Note that, the set $\left\{G^{c}\left(N_{1}\right) \cap G^{c}\left(N_{2}\right) \cap \cdots \cap G^{c}\left(N_{k}\right) \mid N_{i} \in M, 1 \leq i \leq k, k \in \mathbb{N}\right\}$ is a basis for the Zariski-like topology of $M$.

Let $M$ be a lattice module over a $C$-lattice $L$ and let $\operatorname{Spec}^{c p}(M)$ be equipped with the Zariski-like topology. Let $Y \subseteq \operatorname{Spec}^{c p}(M)$. The closure of $Y$ in $\operatorname{Spec}^{c p}(M)$ is denoted by $C l(Y)$ and meet of all elements of $Y$ by $Z(Y)$. Note that, if $Y=\emptyset$, then $Z(Y)=1_{M}$.

Lemma 2.2. Let $M$ be a lattice module over a $C$-lattice $L$ and let $Y$ be a non-empty subset of $\operatorname{Spec}^{c p}(M)$. Then $C l(Y)=\cup_{P \in Y} F^{c}(P)$.

Proof. Suppose that $Y$ is a non-empty subset of $\operatorname{Spec}^{c p}(M)$. Clearly $Y \subseteq \cup_{P \in Y} F^{c}(P)$. Suppose $D$ is any closed subset of $\operatorname{Spec}^{c p}(M)$ such that $Y \subseteq D$. Thus $D=$ $\cap_{k \in J}\left(\cup_{l=1}^{n_{k}} F^{c}\left(N_{k l}\right)\right)$, for some $N_{k l} \in M, k \in J$ and $n_{k} \in \mathbb{N}$. Let $P_{1} \in \cup_{P \in Y} F^{c}(P)$, then there exists $P_{0} \in Y$ such that $P_{1} \in F^{c}\left(P_{0}\right)$ and so $P_{0} \leq P_{1}$. Since $P_{0} \in Y \subseteq D$, therefore for each $k \in J$ there exists $l \in\left\{1,2, \cdots, n_{k}\right\}$ such that $N_{k l} \leq P_{0}$ and hence $N_{k l} \leq P_{0} \leq P_{1}$. Therefore $P_{1} \in F^{c}\left(N_{k l}\right)$ for each $k \in J$ and hence $P_{1} \in \cap_{k \in J}\left(\cup_{l=1}^{n_{k}} F^{c}\left(N_{k l}\right)\right)=D$. It follows that $\cup_{P \in Y} F^{c}(P) \subseteq D$. Thus $\cup_{P \in Y} F^{c}(P)$ is the smallest closed set in $\operatorname{Spec}^{c p}(M)$ which contains $Y$. Consequently, $C l(Y)=$ $\cup_{P \in Y} F^{c}(P)$.

Corollary 2.3. Let $M$ be a lattice module over a C-lattice L. Then

(1) $C l(\{P\})=F^{c}(P)$, for each $P \in \operatorname{Spec}^{c p}(M)$.

(2) $P_{0} \in C l(\{P\})$ if and only if $P \leq P_{0}$ if and only if $F^{c}\left(P_{0}\right) \subseteq F^{c}(P)$, for $P_{0} \in \operatorname{Spec}^{c p}(M)$.

(3) The set $\{P\}$ is closed in $\operatorname{Spec}^{c p}(M)$ if and only if $P$ is a maximal classical prime element of $M$.

Proof. (1) By Lemma 2.2, for $Y \subseteq \operatorname{Spec}^{c p}(M), C l(Y)=\cup_{P \in Y} F^{c}(P)$. Assume that $Y=\{P\}$, then $\cup_{P \in Y} F^{c}(P)=F^{c}(P)$. Hence $C l(\{P\})=F^{c}(P)$.

(2) Suppose that $P_{0} \in C l(\{P\})$. Then $P_{0} \in C l(\{P\})=F^{c}(P)$, by part (1) and 
therefore $P \leq P_{0}$ which implies that $F^{c}\left(P_{0}\right) \subseteq F^{c}(P)$. Conversely, suppose that $F^{c}\left(P_{0}\right) \subseteq F^{c}(P)$. Since $P_{0} \in F^{c}\left(P_{0}\right) \subseteq F^{c}(P), P \leq P_{0}$ and hence $P_{0} \in F^{c}(P)=$ $C l(\{P\})$.

(3) Suppose that the set $\{P\}$ is closed in $\operatorname{Spec}^{c p}(M)$ and $P$ is not maximal, then there exists $Q$ such that $P \leq Q$, which implies that $Q \in C l(\{P\})$ by part (2). But $\{P\}$ is closed, therefore $Q \in\{P\}$ and so $P=Q$. Consequently, $P$ is a maximal classical prime element. Conversely, suppose that $P$ is a maximal classical prime element of $M$. Let $Q \in C l(\{P\})$. Then by part $(1), Q \in F^{c}(P)$, therefore $P \leq Q$. But $P$ is maximal, hence $P=Q$ and therefore $C l(\{P\})=\{P\}$. Consequently, $\{\mathrm{P}\}$ is closed in $\operatorname{Spec}^{c p}(M)$.

Lemma 2.4. Let $M$ be a lattice module over a $C$-lattice $L$ and let $Y$ be a non-empty closed subset of $\operatorname{Spec}^{c p}(M)$, then $Y=\cup_{P \in Y} F^{c}(P)$.

Proof. Let $Y$ be a non-empty closed subset of $\operatorname{Spec}^{c p}(M)$. It is clear that, $Y \subseteq$ $\cup_{P \in Y} F^{c}(P)$. By Corollary 2.3(1), for each $P \in Y, C l(\{P\})=F^{c}(P)$, therefore $F^{c}(P)=C l(\{P\}) \subseteq C l(Y)=Y$. Hence $\cup_{P \in Y} F^{c}(P) \subseteq Y$. Consequently, $Y=$ $\cup_{P \in Y} F^{c}(P)$.

A topological space $X$ is called irreducible if $X \neq \emptyset$ and every finite intersection of non-empty open sets of $X$ is non-empty. A non-empty subset $Y$ of a topological space $X$ is called an irreducible set if the subspace $Y$ of $X$ is irreducible, equivalently, for any two closed sets $Y_{1}$ and $Y_{2}$ of $X, Y \subseteq Y_{1} \cup Y_{2}$ implies either $Y \subseteq Y_{1}$ or $Y \subseteq Y_{2}$ ([8]).

Lemma 2.5. Let $M$ be a lattice module over a $C$-lattice $L$. Then for each $P \in$ $\operatorname{Spec}^{c p}(M), F^{c}(P)$ is irreducible.

Proof. Suppose that $X_{1}$ and $X_{2}$ are two closed subsets of $\operatorname{Spec}^{c p}(M)$ and $F^{c}(P) \subseteq$ $X_{1} \cup X_{2}$. Since $P \in F^{c}(P)$, therefore $P \in X_{1} \cup X_{2}$ which implies either $P \in X_{1}$ or $P \in X_{2}$. Suppose that $P \in X_{1}$. Since $X_{1}$ is closed in $\operatorname{Spec}^{c p}(M)$, we have $X_{1}=$ $\cap_{k \in J}\left(\cup_{l=1}^{n_{k}} F^{c}\left(N_{k l}\right)\right)$, for some $N_{k l} \in M, k \in J, n_{k} \in \mathbb{N}$. Thus $P \in \cup_{l=1}^{n_{k}} F^{c}\left(N_{k l}\right)$, for each $k \in J$. It follows that $F^{c}(P) \subseteq \cup_{l=1}^{n_{k}} F^{c}\left(N_{k l}\right)$, for each $k \in J$. Therefore $F^{c}(P) \subseteq \cap_{k \in J}\left(\cup_{l=1}^{n_{k}} F^{c}\left(N_{k l}\right)\right)=X_{1}$. Consequently, $F^{c}(P)$ is irreducible.

Theorem 2.6. Let $M$ be a lattice module over a $C$-lattice $L$ and $Y \subseteq \operatorname{Spec}^{c p}(M)$. Then

(1) If $Y$ is irreducible, then $Z(Y)$ is a classical prime element.

(2) If $Z(Y)$ is a classical prime element and $Z(Y) \in C l(Y)$, then $Y$ is irreducible. 
Proof. (1) Suppose that $Y$ is an irreducible subset of $\operatorname{Spec}^{c p}(M)$. Clearly, $Z(Y)=$ $\wedge_{P \in Y} P<1_{M}$ and $Y \subseteq F^{c}(Z(Y))$. Let $a b X \leq Z(Y)$, for $a, b \in L$ and $X \in M$. Now for $P \in Y, P \in F^{c}(Z(Y))$, hence $Z(Y) \leq P$ and therefore $a b X \leq Z(Y) \leq P$. Since $P$ is classical prime, either $a X \leq P$ or $b X \leq P$, which implies that $P \in F^{c}(a X)$ or $P \in F^{c}(b X)$ and hence $P \in F^{c}(a X) \cup F^{c}(b X)$. Therefore $Y \subseteq F^{c}(a X) \cup F^{c}(b X)$. Since $Y$ is irreducible, $Y \subseteq F^{c}(a X)$ or $Y \subseteq F^{c}(b X)$. If $Y \subseteq F^{c}(a X)$, then $a X \leq P$, for all $P \in Y$ and hence $a X \leq Z(Y)$. If $Y \subseteq F^{c}(b X)$, then $b X \leq P$, for all $P \in Y$, hence $b X \leq Z(Y)$. Consequently, $\mathrm{Z}(\mathrm{Y})$ is a classical prime element of $M$.

(2) Suppose that $Z(Y)$ is a classical prime element and $Z(Y) \in C l(Y)$. We have $Z(Y) \leq P$, for each $P \in Y$, therefore $F^{c}(P) \subseteq F^{c}(Z(Y))$, for each $P \in Y$ by Corollary 2.3(2). Thus $C l(Y)=\cup_{P \in Y} F^{c}(P) \subseteq F^{c}(Z(Y))$, by Lemma 2.2. On the other hand, since $Z(Y)$ is a classical prime element and $Z(Y) \in C l(Y), F^{c}(Z(Y)) \subseteq$ $C l(Y)$. Consequently, $C l(Y)=F^{c}(Z(Y))$. Now suppose that $Y \subseteq Y_{1} \cup Y_{2}$, where $Y_{1}$ and $Y_{2}$ are closed subsets of $\operatorname{Spec}^{c p}(M)$, then $C l(Y) \subseteq Y_{1} \cup Y_{2}$ and hence $F^{c}(Z(Y)) \subseteq Y_{1} \cup Y_{2}$. Since $Z(Y)$ is a classical prime element, $F^{c}(Z(Y))$ is irreducible by Lemma 2.5. Therefore we have $F^{c}(Z(Y)) \subseteq Y_{1}$ or $F^{c}(Z(Y)) \subseteq Y_{2}$. It follows that $Y \subseteq Y_{1}$ or $Y \subseteq Y_{2}$. Consequently, $Y$ is irreducible.

Definition 2.7. Let $M$ be a lattice module over a $C$-lattice $L$. Let $N$ be an element of $M$. Then the classical prime radical $\sqrt[c p]{N}$ of $N$ is the meet of all classical prime elements of $M$ containing $N$, i.e., $\sqrt[c p]{N}=\wedge\left\{P \in \operatorname{Spec}^{c p}(M) \mid N \leq P\right\}$.

$\sqrt[c p]{N}=1_{M}$, if there is no classical prime element which contains $N$. An element $N$ is said to be classical prime radical element if $N=\sqrt[c p]{N}$. Note that, $N \leq \sqrt[c p]{N}$ and $F^{c}(N)=F^{c}(\sqrt[c p]{N})$.

Corollary 2.8. Let $M$ be a lattice module over a $C$-lattice $L$ and let $N$ be any element of $M$. Then the following are equivalent:

(1) The subset $F^{c}(N)$ of $\operatorname{Spec}^{c p}(M)$ is irreducible.

(2) $\sqrt[c p]{N}$ is a classical prime element.

Proof. (1) $\Rightarrow(2)$ Suppose that $F^{c}(N)$ is an irreducible subset of $\operatorname{Spec}^{c p}(M)$, then $Z\left(F^{c}(N)\right)$ is classical prime element of $M$, by Theorem 2.6(1). Now, $\sqrt[c p]{N}=\wedge\{P \in$ $\left.\operatorname{Spec}^{c p}(M) \mid N \leq P\right\}=\wedge\left\{P \in F^{c}(N)\right\}=Z\left(F^{c}(N)\right)$. Consequently, $\sqrt[c p]{N}$ is a classical prime element.

$(2) \Rightarrow(1)$ Suppose that $\sqrt[c p]{N}$ is a classical prime element, then $F^{c}(\sqrt[c p]{N})$ is irreducible by Lemma 2.5. Since, for each $N \in M, F^{c}(N)=F^{c}(\sqrt[c p]{N})$, therefore $F^{c}(N)$ is an irreducible subset of $\operatorname{Spec}^{c p}(M)$. 
Let $Y$ be a closed subset of a topological space. An element $y \in Y$ is called a generic point of $Y$ if $Y=C l(\{y\})$ (see [3]). Note that, a generic point of the irreducible closed subset $Y$ of a topological space is unique if the topological space is a $T_{0}$-space.

Theorem 2.9. Let $M$ be a lattice module over a $C$-lattice $L$. Then

(1) $\operatorname{Spec}^{c p}(M)$ is always a $T_{0}$-space.

(2) Every $P \in \operatorname{Spec}^{c p}(M)$ is a generic point of the irreducible closed subset $F^{c}(P)$.

(3) Every finite irreducible closed subset of $\operatorname{Spec}^{c p}(M)$ has a generic point.

Proof. (1) Suppose that $P_{1}, P_{2} \in \operatorname{Spec}^{c p}(M)$. Then by Corollary 2.3(1), $C l\left(\left\{P_{1}\right\}\right)=$ $F^{c}\left(P_{1}\right), C l\left(\left\{P_{2}\right\}\right)=F^{c}\left(P_{2}\right)$ and therefore $C l\left(\left\{P_{1}\right\}\right)=C l\left(\left\{P_{2}\right\}\right)$ if and only if $F^{c}\left(P_{1}\right)=F^{c}\left(P_{2}\right)$ if and only if $P_{1}=P_{2}$ by Corollary 2.3(2). Now, by the fact that a topological space is a $T_{0}$-space if the closures of distinct points are distinct, we conclude that, $\operatorname{Spec}^{c p}(M)$ is a $T_{0}$-space.

(2) For each $P \in \operatorname{Spec}^{c p}(M), F^{c}(P)=C l(\{P\})$ by Corollary 2.3(1). Hence $P$ is a generic point of the irreducible closed subset $F^{c}(P)$.

(3) Suppose that $Y$ is an irreducible closed subset of $\operatorname{Spec}^{c p}(M)$ and $Y=\left\{P_{1}, P_{2}, \cdots, P_{k}\right\}$, where $P_{i} \in \operatorname{Spec}^{c p}(M), k \in \mathbb{N}$. By Lemma 2.4, $Y=$ $C l(Y)=F^{c}\left(P_{1}\right) \cup F^{c}\left(P_{2}\right) \cup \cdots \cup F^{c}\left(P_{k}\right)$. Since $Y$ is irreducible, $Y=F^{c}\left(P_{i}\right)$, for some $i(1 \leq i \leq k)$. By part (2), $P_{i}$ is a generic point of $F^{c}\left(P_{i}\right)=Y$.

Theorem 2.10. Let $M$ be a lattice module over a $C$-lattice $L$ such that $M$ has ascending chain condition on classical prime radical elements. Then $\operatorname{Spec}^{c p}(M)$ with the Zariski-like topology is a quasi-compact space.

Proof. Let $M$ be a lattice module over a $C$-lattice $L$ and suppose that $M$ has ascending chain condition on classical prime radical elements. Let $\mathcal{B}$ be a family of open sets covering $\operatorname{Spec}^{c p}(M)$ and suppose that no finite subfamily of $\mathcal{B}$ covers $\operatorname{Spec}^{c p}(M)$. Since $F^{c}\left(\sqrt[c p]{0_{M}}\right)=F^{c}\left(0_{M}\right)=\operatorname{Spec}^{c p}(M)$, we may use the ascending chain condition on classical prime radical elements to choose an element $N$ maximal with respect to the property that no finite subfamily of $\mathcal{B}$ covers $F^{c}(N)$ (we may assume $N=\sqrt[c p]{N}$, because $\left.F^{c}(N)=F^{c}(\sqrt[c p]{N})\right)$

Suppose that $N$ is not classical prime element of $M$. Then there exists $X \in M$ and $a, b \in L$, such that $a b X \leq N, a X \not \leq N$ and $b X \not \leq N$. Thus $N<N \vee a X \leq$ $\sqrt[c p]{N \vee a X}$ and $N<N \vee b X \leq \sqrt[c p]{N \vee b X}$. Hence, without loss of generality, there must exists a finite subfamily $\mathcal{B}^{\prime}$ of $\mathcal{B}$ that covers both $F^{c}(N \vee a X)$ and $F^{c}(N \vee b X)$. Let $P \in F^{c}(N)$. Since $a b X \leq N$, therefore $a b X \leq P$ and since $P$ is classical prime, we have $a X \leq P$ or $b X \leq P$. Thus $P \in F^{c}(N \vee a X)$ or $P \in F^{c}(N \vee b X)$ and 
therefore $F^{c}(N) \subseteq F^{c}(N \vee a X) \cup F^{c}(N \vee b X)$. Thus $F^{c}(N)$ is covered with the finite subfamily $\mathcal{B}^{\prime}$, which is contradiction. Therefore $N$ is a classical prime element of $M$.

Now choose $U \in \mathcal{B}$ such that $N \in U$. Thus $N$ must have a neighborhood $\cap_{i=1}^{n} G^{c}\left(K_{i}\right)$, for some $K_{i} \in M$ and $n \in \mathbb{N}$, such that $\cap_{i=1}^{n} G^{c}\left(K_{i}\right) \subseteq U$. Suppose that for each $i(1 \leq i \leq n), P \in G^{c}\left(K_{i} \vee N\right) \cap F^{c}(N)$, then $K_{i} \vee N \not \leq P$ and $N \leq P$. Thus $K_{i} \not \leq P$ and $P \in G^{c}\left(K_{i}\right)$. Consequently, $N \in\left[G^{c}\left(K_{i} \vee N\right) \cap F^{c}(N)\right] \subseteq G^{c}\left(K_{i}\right)$ and hence for each $i(1 \leq i \leq n), N \in \cap_{i=1}^{n}\left[G^{c}\left(K_{i} \vee N\right) \cap F^{c}(N)\right] \subseteq \cap_{i=1}^{n} G^{c}\left(K_{i}\right) \subseteq U$. Thus $\left[\cap_{i=1}^{n} G^{c}\left(K_{i} \vee N\right)\right] \cap F^{c}(N)$, where $N<K_{i} \vee N$ is a neighborhood of $N$, with $\left[\cap_{i=1}^{n} G^{c}\left(K_{i} \vee N\right)\right] \cap F^{c}(N) \subseteq U$.

Since for each $i(1 \leq i \leq n), N<K_{i} \vee N, F^{c}\left(K_{i} \vee N\right)$ can be covered by some finite subfamily $\mathcal{B}_{i}^{\prime}$ of $\mathcal{B}$. Now $F^{c}(N)-\left[\cup_{i=1}^{n} F^{c}\left(K_{i} \vee N\right)\right]=F^{c}(N)-\left[\cap_{i=1}^{n} G^{c}\left(K_{i} \vee N\right)\right]^{\prime}=$ $\cap_{i=1}^{n} G^{c}\left(K_{i} \vee N\right) \cap F^{c}(N) \subseteq U$ (here' denotes complement). Therefore $F^{c}(N)$ can be covered by $\mathcal{B}_{1}^{\prime} \cup \mathcal{B}_{2}^{\prime} \cup \cdots \cup \mathcal{B}_{n}^{\prime} \cup\{U\}$, which is contradiction to our choice of $N$. Thus there must exists a finite subfamily of $\mathcal{B}$ which covers $\operatorname{Spec}^{c p}(M)$. Therefore, $\operatorname{Spec}^{c p}(M)$ is a quasi-compact space.

A topological space $X$ is a spectral space if $X$ is homeomorphic to $\operatorname{Spec}(S)$, with Zariski topology, for some commutative ring $S$. Spectral spaces have been characterized by Hochster $([12])$ as the topological spaces $X$ which satisfy the following conditions.

(1) $X$ is a $T_{0}$-space.

(2) $X$ is a quasi-compact.

(3) The quasi-compact open subsets of $X$ are closed under finite intersection and form an open basis.

(4) Each irreducible closed subset of $X$ has a generic point.

Theorem 2.11. Let $M$ be a lattice module over a $C$-lattice $L$ with finite spectrum. Then $\operatorname{Spec}^{c p}(M)$ is a spectral space.

Proof. Since $\operatorname{Spec}^{c p}(M)$ is finite, by Theorem 2.9, $\operatorname{Spec}^{c p}(M)$ is a $T_{0}$-space and every irreducible closed subset of $\operatorname{Spec}^{c p}(M)$ has a generic point. Also, since $\operatorname{Spec}^{c p}(M)$ is finite, it is quasi-compact and the quasi-compact open subsets of $\operatorname{Spec}^{c p}(M)$ are closed under finite intersections and form an open basis ([9]). Hence, by Hochster's characterization, $\operatorname{Spec}^{c p}(M)$ is a spectral space.

\section{Patch-like topology on $\operatorname{Spec}^{c p}(M)$}

Let $X$ be a topological space. By the patch topology on $X$ we mean the topology which has as a sub-basis for its closed sets the closed sets and compact open sets 
of the original space. By a patch we mean set closed in the patch topology ([11], $[12])$.

Definition 3.1. Let $M$ be a lattice module over a $C$-lattice $L$. Let $E(M)$ be the family of all subsets of $\operatorname{Spec}^{c p}(M)$ of the form $F^{c}(N) \cap G^{c}(K)$, where $N, K \in M$. Clearly $E(M)$ contains both $\operatorname{Spec}^{c p}(M)=F^{c}\left(0_{M}\right) \cap G^{c}\left(1_{M}\right)$ and empty set $\emptyset=$ $F^{c}\left(1_{M}\right) \cap G^{c}\left(0_{M}\right)$. Let $T_{p}(M)$ be the collection $U$ of all unions of finite intersections of elements of $E(M)$. Then $T_{p}(M)$ is a topology on $\operatorname{Spec}^{c p}(M)$ and is called the patch-like topology of $M$. In fact $E(M)$ is a sub-basis for the patch-like topology of $M$.

Note that, the patch-like topology on $\operatorname{Spec}^{c p}(M)$ is finer than the Zariski-like topology on $\operatorname{Spec}^{c p}(M)$.

Theorem 3.2. Let $M$ be a lattice module over a C-lattice L. Then $\operatorname{Spec}^{c p}(M)$ with the patch-like topology is a Hausdorff space.

Proof. Suppose that $P_{1}, P_{2} \in \operatorname{Spec}^{c p}(M)$ and $P_{1} \neq P_{2}$. Since $P_{1} \neq P_{2}$, so either $P_{1} \not \leq P_{2}$ or $P_{2} \not \leq P_{1}$. Suppose that $P_{1} \not \leq P_{2}$. By Definition $3.1, U_{1}=G^{c}\left(1_{M}\right) \cap$ $F^{c}\left(P_{1}\right)$ is a patch-like-neighborhood of $P_{1}$ and $U_{2}=G^{c}\left(P_{1}\right) \cap F^{c}\left(P_{2}\right)$ is a patchlike-neighborhood of $P_{2}$. Clearly, $G^{c}\left(P_{1}\right) \cap F^{c}\left(P_{1}\right)=\emptyset$ and hence $U_{1} \cap U_{2}=\emptyset$. Therefore, $\operatorname{Spec}^{c p}(M)$ is a Hausdorff space.

Theorem 3.3. Let $M$ be a lattice module over a $C$-lattice $L$ such that $M$ has ascending chain condition on classical prime radical elements. Then $\operatorname{Spec}^{c p}(M)$ with the patch-like topology is a compact space.

Proof. Let $M$ be a lattice module over a $C$-lattice $L$ and suppose that $M$ has ascending chain condition on classical prime radical elements. Let $\mathcal{A}$ be a family of open sets covering $\operatorname{Spec}^{c p}(M)$ and suppose that no finite subfamily of $\mathcal{A}$ covers $\operatorname{Spec}^{c p}(M)$. Since $F^{c}\left(\sqrt[c p]{0_{M}}\right)=F^{c}\left(0_{M}\right)=\operatorname{Spec}^{c p}(M)$, we may use the ascending chain condition on classical prime radical elements to choose an element $N$ maximal with respect to the property that no finite subfamily of $\mathcal{A}$ covers $F^{c}(N)$ (we may assume $N=\sqrt[c p]{N}$, because $\left.F^{c}(N)=F^{c}(\sqrt[c p]{N})\right)$.

Suppose that $N$ is not classical prime element of $M$. Then there exists $X \in M$ and $a, b \in L$, such that $a b X \leq N, a X \not \leq N$ and $b X \not \leq N$. Thus $N<N \vee a X \leq$ $\sqrt[c p]{N \vee a X}$ and $N<N \vee b X \leq \sqrt[c p]{N \vee b X}$. Hence, without loss of generality, there must exists a finite subfamily $\mathcal{A}^{\prime}$ of $\mathcal{A}$ that covers both $F^{c}(N \vee a X)$ and $F^{c}(N \vee b X)$. Let $P \in F^{c}(N)$. Since $a b X \leq N$ and $N \leq P$, we have $a b X \leq P$. Since $P$ is classical prime, we have either $a X \leq P$ or $b X \leq P$. Thus $N \vee a X \leq P$ or $N \vee b X \leq P$. Therefore, either $P \in F^{c}(N \vee a X)$ or $P \in F^{c}(N \vee b X)$ and 
hence $F^{c}(N) \subseteq F^{c}(N \vee a X) \cup F^{c}(N \vee b X)$. Thus $F^{c}(N)$ is covered with the finite subfamily $\mathcal{A}^{\prime}$, which is contradiction. Therefore $N$ is a classical prime element of $M$.

Now choose $U \in \mathcal{A}$ such that $N \in U$. Thus $N$ must have a patch-like-neighborhood $\cap_{i=1}^{n}\left[G^{c}\left(K_{i}\right) \cap F^{c}\left(N_{i}\right)\right]$ for some $K_{i}, N_{i} \in M, n \in \mathbb{N}$ such that $\cap_{i=1}^{n}\left[G^{c}\left(K_{i}\right) \cap\right.$ $\left.F^{c}\left(N_{i}\right)\right] \subseteq U$. Suppose that for each $i(1 \leq i \leq n), P \in\left[G^{c}\left(K_{i} \vee N\right) \cap F^{c}(N)\right]$. Then $P \in G^{c}\left(K_{i} \vee N\right)$ and $P \in F^{c}(N)$ and so that $K_{i} \vee N \not \leq P$ and $N \leq P$. Thus $K_{i} \not \leq P$, i.e., $P \in G^{c}\left(K_{i}\right)$. On the other hand, $N \in F^{c}\left(N_{i}\right)$, therefore $N_{i} \leq N$ and $N_{i} \leq N, N \leq P$ implies $N_{i} \leq P$, hence $P \in F^{c}\left(N_{i}\right)$. Consequently, $N \in\left[G^{c}\left(K_{i} \vee N\right) \cap F^{c}(N)\right] \subseteq\left[G^{c}\left(K_{i}\right) \cap F^{c}\left(N_{i}\right)\right]$ and hence $N \in \cap_{i=1}^{n}\left[G^{c}\left(K_{i} \vee N\right) \cap\right.$ $\left.F^{c}(N)\right] \subseteq \cap_{i=1}^{n}\left[G^{c}\left(K_{i}\right) \cap F^{c}\left(N_{i}\right)\right] \subseteq U$. Thus $\left[\cap_{i=1}^{n} G^{c}\left(K_{i} \vee N\right)\right] \cap F^{c}(N)$, where $N<K_{i} \vee N$, is a neighborhood of $N$, with $\left[\cap_{i=1}^{n} G^{c}\left(K_{i} \vee N\right)\right] \cap F^{c}(N) \subseteq U$.

Since for each $i(1 \leq i \leq n), N<K_{i} \vee N, F^{c}\left(K_{i} \vee N\right)$ is covered by some finite subfamily $\mathcal{A}_{i}^{\prime}$ of $\mathcal{A}$. Now $F^{c}(N)-\left[\cup_{i=1}^{n} F^{c}\left(K_{i} \vee N\right)\right]=F^{c}(N)-\left[\cap_{i=1}^{n} G^{c}\left(K_{i} \vee N\right)\right]^{\prime}=$ $\left[\cap_{i=1}^{n} G^{c}\left(K_{i} \vee N\right)\right] \cap F^{c}(N) \subseteq U$ (here' denotes complement). Hence $F^{c}(N)$ can be covered by $\mathcal{A}_{1}^{\prime} \cup \mathcal{A}_{2}^{\prime} \cup \cdots \cup \mathcal{A}_{n}^{\prime} \cup\{U\}$, which is contradiction to our choice of $N$. Thus there must exists a finite subfamily of $\mathcal{A}$ which covers $\operatorname{Spec}^{c p}(M)$. Therefore, $\operatorname{Spec}^{c p}(M)$ is compact in the patch-like topology of $M$.

We require the following evident Lemma.

Lemma 3.4. Let $\tau_{1}$ and $\tau_{2}$ be two topologies on $X$ such that $\tau_{1} \subseteq \tau_{2}$. If $X$ is quasi-compact (i.e. any open cover of it has a finite subcover) in $\tau_{2}$, then $X$ is also quasi-compact in $\tau_{1}$.

Theorem 3.5. Let $M$ be a lattice module over a $C$-lattice $L$ such that $M$ has ascending chain condition on classical prime radical elements. Then for each $n \in \mathbb{N}$ and elements $N_{i}(1 \leq i \leq n)$ of $M, G^{c}\left(N_{1}\right) \cap G^{c}\left(N_{2}\right) \cap \cdots \cap G^{c}\left(N_{n}\right)$ is a quasi-compact subset of $\operatorname{Spec}^{c p}(M)$ with the Zariski-like topology. Consequently, Spec ${ }^{c p}(M)$ has a basis of quasi-compact open subsets.

Proof. By Definition 3.1, for each element $N$ of $M, F^{c}(N)=F^{c}(N) \cap G^{c}\left(1_{M}\right)$ is an open subset of $\operatorname{Spec}^{c p}(M)$ with the patch-like topology and $G^{c}(N)$ is a complement of $F^{c}(N)$. Therefore for each $N \in M, G^{c}(N)$ is a closed subset in $\operatorname{Spec}^{c p}(M)$. Thus for each $n \in \mathbb{N}$ and elements $N_{i}(1 \leq i \leq n)$ of $M, G^{c}\left(N_{1}\right) \cap G^{c}\left(N_{2}\right) \cap \cdots \cap G^{c}\left(N_{n}\right)$ is also a closed subset in $\operatorname{Spec}^{c p}(M)$ with the patch-like topology. Since every closed subset of a compact space is compact, therefore $G^{c}\left(N_{1}\right) \cap G^{c}\left(N_{2}\right) \cap \cdots \cap G^{c}\left(N_{n}\right)$ is compact in $\operatorname{Spec}^{c p}(M)$ with the patch-like topology and by Lemma 3.4, it is quasicompact in $\operatorname{Spec}^{c p}(M)$ with the Zariski-like topology. Now, $\operatorname{Spec}^{c p}(M)=G^{c}\left(1_{M}\right)$ and $\mathbb{B}=\left\{G^{c}\left(N_{1}\right) \cap G^{c}\left(N_{2}\right) \cap \cdots \cap G^{c}\left(N_{n}\right) \mid N_{i} \in M, 1 \leq i \leq n, n \in \mathbb{N}\right\}$ is a basis 
for the Zariski-like topology of $M$. Consequently, $\operatorname{Spec}^{c p}(M)$ is quasi-compact and has a basis of quasi-compact open subsets.

Corollary 3.6. Let $M$ be a lattice module over a $C$-lattice $L$ such that $M$ has ascending chain condition on classical prime radical elements. Then quasi-compact open sets of $\operatorname{Spec}^{c p}(M)$ (with the Zariski-like topology) are closed under finite intersections.

Proof. Let $U_{1}$ and $U_{2}$ be two quasi-compact open sets of $\operatorname{Spec}^{c p}(M)$ and let $U=$ $U_{1} \cap U_{2}$. Each of $U_{1}$ and $U_{2}$ is a finite union of members of $\mathbb{B}=\left\{G^{c}\left(N_{1}\right) \cap G^{c}\left(N_{2}\right) \cap\right.$ $\left.\cdots \cap G^{c}\left(N_{n}\right) \mid N_{i} \in M, 1 \leq i \leq n, n \in \mathbb{N}\right\}$ and hence $U=\cup_{i=1}^{m}\left(\cap_{j=1}^{n_{i}} G^{c}\left(N_{j}\right)\right)$. Let $\Pi$ be any open cover of $U$. Then $\Pi$ also covers each $\cap_{j=1}^{n_{i}} G^{c}\left(N_{j}\right)$ which is quasi-compact by Theorem 3.5. Hence each $\cap_{j=1}^{n_{i}} G^{c}\left(N_{j}\right)$ has a finite subcover of $\Pi$ and therefore $U$ has also a finite subcover of $\Pi$. Thus $U$ is quasi-compact, as required.

Lemma 3.7. Let $M$ be a lattice module over a $C$-lattice $L$ such that $M$ has ascending chain condition on classical prime radical elements. Then every irreducible closed subset of Spec ${ }^{c p}(M)$ (with the Zariski-like topology) has a generic point.

Proof. Suppose that $Y$ is an irreducible closed subset of $\operatorname{Spec}^{c p}(M)$ with the Zariski-like topology. By Lemma 2.4, we have $Y=\cup_{P \in Y} F^{c}(P)$. By Definition 3.1, for each $P \in Y, F^{c}(P)$ is an open subset of $\operatorname{Spec}^{c p}(M)$ with the patch-like topology. Now, since $Y \subseteq \operatorname{Spec}^{c p}(M)$ is closed with the Zariski-like topology, the complement of $Y$ is open by this topology and therefore the complement of $Y$ is open with the patch-like topology. Hence $Y \subseteq \operatorname{Spec}^{c p}(M)$ is closed with the patch-like topology. By Theorem 3.3, $\operatorname{Spec}^{c p}(M)$ is compact with the patch-like topology and since $Y \subseteq \operatorname{Spec}^{c p}(M)$ is closed, therefore $Y$ is also compact. Now, since $Y=\cup_{P \in Y} F^{c}(P)$ and each $F^{c}(P)$ is patch-like open, therefore there exists a finite subset $X$ of $Y$ such that $Y=\cup_{P \in X} F^{c}(P)$. Since $Y$ is irreducible, $Y=F^{c}(P)$ for some $P \in X$. Therefore, we have $Y=F^{c}(P)=C l(\{P\})$ for some $P \in Y$. Consequently, $P$ is a generic point for $Y$.

We conclude this section by proving the main theorem .

Theorem 3.8. Let $M$ be a lattice module over a $C$-lattice $L$ such that $M$ has ascending chain condition on classical prime radical elements. Then $\operatorname{Spec}^{c p}(M)$ with the Zariski-like topology is a spectral space.

Proof. By Theorem 2.9, $\operatorname{Spec}^{c p}(M)$ is a $T_{0}$-space. Since $M$ satisfies ascending chain condition on classical prime radical elements, therefore by Theorem 2.10, 
$\operatorname{Spec}^{c p}(M)$ is quasi-compact. By Theorem 3.5, $\operatorname{Spec}^{c p}(M)$ has a basis of quasicompact open subsets and by Corollary 3.6, the family of quasi-compact open subsets of $\operatorname{Spec}^{c p}(M)$ are closed under finite intersections. Finally, by Lemma 3.7, each irreducible closed subset of $\operatorname{Spec}^{c p}(M)$ has a generic point. Thus, by Hochster's characterization, $\operatorname{Spec}^{c p}(M)$ is a spectral space.

Acknowledgement. The authors would like to thank the referee for the valuable suggestions and comments.

\section{References}

[1] F. Alarcon, D. D. Anderson and C. Jayaram, Some results on abstract commutative ideal theory, Period. Math. Hungar., 30(1) (1995), 1-26.

[2] E. A. Al-Khouja, Maximal elements and prime elements in lattice modules, Damascus Univ. J. Basic Sci., 19(2) (2003), 9-21.

[3] M. F. Atiyah and I. G. MacDonald, Introduction to Commutative Algebra, Addison-Wesley Publishing Co., Reading, Mass.-London-Don Mills, Ont. 1969.

[4] S. Ballal and V. Kharat, Zariski topology on lattice modules, Asian-Eur. J. Math., 8(4) (2015), 1550066 (10 pp).

[5] M. Behboodi and M. R. Haddadi, Classical Zariski topology of modules and spectral spaces I, Int. Electron. J. Algebra, 4 (2008), 104-130.

[6] M. Behboodi and M. R. Haddadi, Classical Zariski topology of modules and spectral spaces II, Int. Electron. J. Algebra, 4 (2008), 131-148.

[7] M. Behboodi and M. J. Noori, Zariski-like topology on the classical prime spectrum of a module, Bull. Iranian Math. Soc., 35(1) (2009), 253-269.

[8] N. Bourbaki, Algebre Commutative, Chap 1-2, Hermann, Paris, 1961.

[9] N. Bourbaki, Elements of Mathematics, General topology, Part 1, Hermann, Paris; Addison-Wesley Publishing Co., Reading, Mass.-London-Don Mills, Ont. 1966.

[10] F. Callialp, G. Ulucak and U. Tekir, On the Zariski topology over an L-module M, Turkish J. Math., 41(2) (2017), 326-336.

[11] K. R. Goodearl and R. B. Warfield, Jr., An Introduction to Noncommutative Noetherian Rings, Second edition, London Math. Soc. Student Texts, 16, Cambridge Univ. Press, Cambridge, 2004.

[12] M. Hochster, Prime ideal structure in commutative rings, Trans. Amer. Math. Soc., 142 (1969), 43-60.

[13] J. A. Johnson, A-adic completions of Noetherian lattice modules, Fund. Math., 66 (1970), 347-373. 
[14] C. P. Lu, The Zariski topology on the prime spectrum of a module, Houston J. Math., 25(3) (1999), 417-432.

[15] C. S. Manjarekar and A. V. Bingi, Absorbing elements in lattice modules, Int. Electron. J. Algebra, 19 (2016), 58-76.

[16] J. R. Munkres, Topology: a First Course, Prentice-Hall, Inc. Eglewood Cliffs, New Jersey, 1975.

[17] N. Phadatare, S. Ballal and V. Kharat, On the second spectrum of lattice modules, Discuss. Math. Gen. Algebra Appl., 37(1) (2017), 59-74.

[18] N. Phadatare and V. Kharat, On the second radical elements of lattice modules, Tbilisi Math. J., 11(4) (2018), 165-173.

[19] N. Phadatare, V. Kharat and S. Ballal, On the maximal spectrum of lattice modules, Southeast Asian Bull. Math., Accepted.

[20] N. K. Thakare and C. S. Manjarekar, Abstract spectral theory: Multiplicative lattices in which every character is contained in a unique maximal character, Algebra and its applications (New Delhi, 1981), Lecture Notes in Pure and Appl. Math., 91, Dekker, New York, (1984), 265-276.

[21] N. K. Thakare, C. S. Manjarekar and S. Maeda, Abstract spectral theory II: minimal characters and minimal spectrums of multiplicative lattices, Acta Sci. Math. (Szeged), 52(1-2) (1988), 53-67.

\section{Pradip Girase}

Department of Mathematics

K. K. M. College, Manwath

Dist-Parbhani(M.S.)-431505, India

e-mail: pgpradipmaths22@gmail.com

Vandeo Borkar (Corresponding Author)

Department of Mathematics

Yeshwant Mahavidyalaya

Nanded(M.S.)-431602, India

e-mail: borkarvc@gmail.com

\section{Narayan Phadatare}

Department of Mathematics

Savitribai Phule Pune University

Pune(M.S.), India

e-mail: a9999phadatare@gmail.com 